\title{
Synergistic effects of colchicine combined with atorvastatin in rats with hyperlipidemia
}

\author{
Congwu Huang ${ }^{1 *}$, Chuan Cen ${ }^{2}$, ChengXu Wang ${ }^{3 *}$, Haiyong Zhan ${ }^{1}$ and Xin Ding ${ }^{2}$
}

\begin{abstract}
Background: Inflammation and endothelial dysfunction is implicated in the atherosclerosis initiation and progression in the setting of hyperlipidemia. Colchicine is a potent anti-inflammatory agent and whether colchicine combined with atorvastatin has synergistic effects on inflammation amelioration and endothelial function improvement is unknown.

Methods: Hyperlipidemic rat model was produced by high-fat and high-cholesterol diet for 6 weeks. Rats with normal diet were served as shame group. In hyperlipidemic group, normal saline, atorvastatin ( $10 \mathrm{mg} / \mathrm{kg}$ body weight/day), colchicines ( $0.5 \mathrm{mg} / \mathrm{kg}$ body weight/day), or atorvastatin combined with colchicines (same dosages) were prescribed for 2 weeks. Serum levels of lipid profile, C-reactive protein (CRP), liver enzyme, lipoprotein associated phospholipase A2 (Lp-PLA2) and nitric oxide (NO) production were serially assessed.
\end{abstract}

Results: Before the beginning of the study, all laboratory variables were comparable among each group. After 6 weeks of hyperlipidemic model production, serum levels of cholesterols, CRP and LP-PLA2 were significantly increased when compared to sham group, whereas NO production was reduced. With 2 weeks of colchicine therapy, serum levels of CRP and Lp-PLA2 were decreased and NO production was enhanced in the colchicine group in a lipid-lowering independent manner. Added colchicine into atorvastatin therapy further improved NO production and decreased CRP and Lp-PLA2 levels, indicating a potential synergism of colchicine and atorvastatin.

Conclusion: Colchicine combined with atorvastatin may have stronger protective effects on improving endothelial function and ameliorating inflammation in rats with hyperlipidemia.

Keywords: Hyperlipidemia, Inflammation, Endothelial dysfunction

\section{Introduction}

Hyperlipidemia is a major cause of multiple diseases such as atherosclerotic cardiovascular diseases (CVD) $[1,2]$. The mechanisms of hyperlipidemia implicated in the initiation and progression of CVD predominantly involve sustained endothelial dysfunction and vascular inflammation [3-5]. Previously, many animal studies and clinical trials also have consistently demonstrated that with statins therapy, a potent agent in regulating lipid metabolism, not only lipid profile disorder has been corrected but also systemic inflammation is

\footnotetext{
*Correspondence: huangcw@vip.sina.com; chengxuwang123@163.com ${ }^{1}$ The Department of Internal Medicine, the Second Affiliated Hospital of Shantou University, Shantou, China

${ }^{3}$ The Emergency Department, the Second Affiliated Hospital of Dalian Medical University, Dalian, China

Full list of author information is available at the end of the article
}

ameliorated as indicated by the decrease of inflammatory cytokines such as C-reactive protein (CRP) [6-9].

Lipoprotein associated phospholipase A2 (Lp-PLA2) is a key enzyme responsible for degrading plateletactivating factor (PAF) and oxidated-LDL (ox-LDL). Initially, some basic studies showed that Lp-PLA2 was beneficial for deterring atherosclerosis progression by means of degrading PAF, a potent pro-inflammatory cytokine [10-12]. Nevertheless, thereafter, a large number of clinical and experimental studies have consistently revealed that increased Lp-PLA2 level was associated with increased risk of cardiovascular events [13-15], which was considered to be associated with the increased production of lyso-phosphotidylcholine (Lyso-PC) and oxidized non-esterified fatty acids (oxNEFAs), two potent proinflammatory and pro-atherosclerotic intermediates derived from ox-LDL degradation by Lp-PLA2 $[16,17]$. 
Notably, some studies showed that statins might have effects on reducing Lp-PLA2 level [18-20], nonetheless, other studies showed no favorable effects of statins on Lp-PLA2 reduction [21,22]. Therefore, whether statins can reduce LP-PLA2 is still inconclusive.

Colchicine is an old medicine and has been used for gout and other inflammatory diseases due to its potent effect on improving inflammatory reactions. Recently, a study conducted by Nidorf and colleagues showed that colchicine combined with statins was beneficial for cardiovascular events' prevention [23]. The underlying mechanisms are far from clear, however. Previously, one study revealed that colchicine could inhibit adhesion of neutrophilic granulocytes to epidermal sections induced by PAF [24]. Since most of circulating Lp-PLA2 is produced by macrophages within vascular wall [17], therefore, we hypothesized that colchicine might reduce Lp-PLA2 production through inhibiting leukocytes adhesion and infiltration.

Taken together, in light of the crucial roles Lp-PLA2 plays on the initiation and progression of vascular inflammation and atherosclerosis in subjects with hyperlipidemia and the potent effect of colchicine on regulating inflammation, we hypothesized that colchicine might be effective in ameliorating vascular inflammation and improving endothelial function by means of declining Lp-PLA2 level, and if corroborated, we believed that in the future adds colchicines into statins therapy may have additional benefits on CVD prevention and therapy.

\section{Methods}

\section{Animal model and study protocol}

Male Sprague-Dawley (SD) rats weighing 200-220 g were obtained from Experimental Animal Center of Shantou University, Shantou, China. The study was approved by Ethic Committee of Shantou University. Totally 50 rats were used in current study and after 1 week's accommodation were evenly and randomly divided into 5 groups. Ten rats given normal diet were served as sham group, and the other 40 rats were given a high-fat and high-cholesterol diet as described by previous study with mild modification (cholesterol 4\%, cholic acid $0.4 \%$, propylthiouracilum $0.3 \%$ and lard $10 \%$ ) for 6 weeks for hyperlipidemic model production [25]. Subsequently, the 40 hyperlipidemic rats were randomly and evenly assigned into 4 groups as follow: control group was orally given normal saline, statins group orally given atorvastatin $(10 \mathrm{mg} / \mathrm{kg}$ body weight/day, reconstituted in normal saline), colchicine group intraperitoneally injected colchicine $(0.5 \mathrm{mg} / \mathrm{kg}$ body weight/day, dissolved in $0.05 \%$ dimethyl sulfoxide, DMSO), and combined group given atorvastatin and colchicine as described above. Total intervention duration was 2 weeks.

\section{Laboratory examination}

Fasting blood was taken for laboratory examination before the beginning of the study, after 6 weeks of model production, and after 2 weeks of intervention. The variables for examination involved serum levels of triglyceride (TG), total cholesterol (TC), low density lipoprotein cholesterol (LDL-C), high density lipoprotein cholesterol (HDL-C), alanine aminotransferase (ALT), aspartate aminotransferase (AST) and CRP, which were assessed by Automatic Biochemistry Analyzer (Hitachi 7150, Tokyo, Japan). Serum level of nitric oxide (NO) was evaluated by nitrite reductase method using Total Nitric Oxide Kit (Beyotime, Haimen, China, S0023), and serum level of LpPLA2 was assessed by sandwich enzyme-linking immunesorbent assay (ELISA) kit (Yanjin Biochemistry Company, Shanghai, China). Three independent experiments were performed in duplicate.

\section{Statistical analyses}

All continuous variables were expressed as mean \pm SD, and analyses were performed with SPSS software, version 18.0 (SPSS Science, Chicago, IL, USA). Statistical significance among groups was evaluated with One Way ANOVA (post hoc LSD-t), and a value of $P<0.05$ was considered statistically significant.

\section{Results}

\section{Changes of lipid profile and other variables}

As presented in Table 1, the baseline laboratory variables among different groups were comparable at the very beginning, and after 6 weeks of high-fat and high-cholesterol diet administration, the serum levels of TG, TC and LDL$\mathrm{C}$ in hyperlipidemic model groups were significantly increased when compared to the sham group. Additionally, serum level of CRP was also profoundly increased in hyperlipidemic model groups, indicating that hyperlipidemia was significantly associated with systemic inflammation. After two weeks' intervention, serum levels of TC and LDL-C in the atorvastatin and combined groups were significantly reduced and no changes were found in the control and colchicine groups. When compared to the atorvastatin group, CRP reduction was more prominent in the colchicine group $(5.35 \pm 0.93 \mathrm{mg} / \mathrm{L}$ versus $4.03 \pm$ $0.65 \mathrm{mg} / \mathrm{L}, \mathrm{P}<0.05)$, indicating that colchicine might have more robust effect on ameliorating inflammation than atorvastatin, which was independent of lipid-lowering. Importantly, this anti-inflammatory effect of colchicine was further enhanced when combined with atorvastatin as evidenced by the magnitude of CRP reduction in the combined group was more prominent than the other groups. Notably, no liver toxicity was found in each group, indicating that current used dosage of atorvastatin and/or colchicine was safe for 2 weeks' therapy in rats. 
Table 1 Changes of lipid profile and other variables $(n=10$, each group)

\begin{tabular}{|c|c|c|c|c|c|}
\hline Variables & Sham & Control & Atorvastatin & Colchicine & Combined \\
\hline \multicolumn{6}{|c|}{ Before the beginning of the study } \\
\hline $\mathrm{TG}(\mathrm{mmol} / \mathrm{L})$ & $0.93 \pm 0.11$ & $0.98 \pm 0.14$ & $0.93 \pm 0.12$ & $0.95 \pm 0.10$ & $0.96 \pm 0.15$ \\
\hline $\mathrm{TC}(\mathrm{mmol} / \mathrm{L})$ & $3.04 \pm 0.35$ & $3.11 \pm 0.32$ & $3.08 \pm 0.27$ & $3.06 \pm 0.30$ & $3.09 \pm 0.33$ \\
\hline LDL-C (mmol/L) & $1.99 \pm 0.24$ & $1.98 \pm 0.21$ & $2.01 \pm 0.29$ & $1.97 \pm 0.19$ & $2.01 \pm 0.24$ \\
\hline $\mathrm{HDL}-\mathrm{C}(\mathrm{mmol} / \mathrm{L})$ & $1.03 \pm 0.08$ & $1.03 \pm 0.07$ & $1.05 \pm 0.07$ & $1.04 \pm 0.06$ & $1.04 \pm 0.05$ \\
\hline $\mathrm{CRP}(\mathrm{mg} / \mathrm{L})$ & $1.24 \pm 0.16$ & $1.27 \pm 0.13$ & $1.32 \pm 0.16$ & $1.25 \pm 0.19$ & $1.24 \pm 0.13$ \\
\hline ALT (U/L) & $24.5 \pm 3.8$ & $25.8 \pm 3.0$ & $25.2 \pm 1.9$ & $24.2 \pm 1.7$ & $26.0 \pm 2.8$ \\
\hline AST (U/L) & $28.3 \pm 2.3$ & $27.6 \pm 2.7$ & $28.2 \pm 2.4$ & $29.1 \pm 3.2$ & $28.8 \pm 2.5$ \\
\hline \multicolumn{6}{|c|}{6 weeks of model production } \\
\hline TG (mmol/L) & $0.98 \pm 0.13^{*}$ & $2.15 \pm 0.33$ & $2.22 \pm 0.37$ & $2.19 \pm 0.28$ & $2.20 \pm 0.27$ \\
\hline $\mathrm{TC}(\mathrm{mmol} / \mathrm{L})$ & $3.08 \pm 0.32^{*}$ & $5.87 \pm 0.66$ & $5.93 \pm 0.61$ & $5.91 \pm 0.57$ & $5.89 \pm 0.60$ \\
\hline LDL-C (mmol/L) & $1.97 \pm 0.21^{*}$ & $3.76 \pm 0.55$ & $3.79 \pm 0.53$ & $3.80 \pm 0.51$ & $3.78 \pm 0.52$ \\
\hline HDL-C (mmol/L) & $1.05 \pm 0.06$ & $1.07 \pm 0.04$ & $1.08 \pm 0.02$ & $1.07 \pm 0.04$ & $1.06 \pm 0.03$ \\
\hline CRP (mg/L) & $1.30 \pm 0.13^{*}$ & $7.25 \pm 1.08$ & $7.20 \pm 1.03$ & $7.18 \pm 1.08$ & $7.22 \pm 1.05$ \\
\hline ALT (U/L) & $27.8 \pm 3.2$ & $29.2 \pm 3.6$ & $28.5 \pm 2.4$ & $26.2 \pm 1.4$ & $26.8 \pm 2.0$ \\
\hline AST (U/L) & $28.2 \pm 3.5$ & $28.2 \pm 2.5$ & $28.7 \pm 2.0$ & $28.3 \pm 2.7$ & $27.4 \pm 1.9$ \\
\hline \multicolumn{6}{|c|}{2 weeks of therapy } \\
\hline TG (mmol/L) & $0.98 \pm 0.13^{*}$ & $2.13 \pm 0.25$ & $1.98 \pm 0.16$ & $2.11 \pm 0.22$ & $1.97 \pm 0.16$ \\
\hline $\mathrm{TC}(\mathrm{mmol} / \mathrm{L})$ & $3.05 \pm 0.24^{*}$ & $5.65 \pm 0.48^{\#}$ & $4.85 \pm 0.41$ & $5.85 \pm 0.55^{\&}$ & $4.64 \pm 0.43$ \\
\hline LDL-C (mmol/L) & $1.93 \pm 0.20^{*}$ & $3.70 \pm 0.51^{\#}$ & $2.92 \pm 0.22$ & $3.71 \pm 0.38^{\&}$ & $2.88 \pm 0.25$ \\
\hline HDL-C (mmol/L) & $1.05 \pm 0.03$ & $1.06 \pm 0.05$ & $1.11 \pm 0.03$ & $1.08 \pm 0.03$ & $1.12 \pm 0.03$ \\
\hline $\mathrm{CRP}(\mathrm{mg} / \mathrm{L})$ & $1.32 \pm 0.12^{*}$ & $7.19 \pm 1.02^{\#}$ & $5.35 \pm 0.93$ & $4.03 \pm 0.65^{\&}$ & $2.87 \pm 0.45^{*}$ \\
\hline ALT (U/L) & $26.3 \pm 4.1$ & $27.8 \pm 1.4$ & $28.2 \pm 2.6$ & $27.4 \pm 1.8$ & $26.3 \pm 2.5$ \\
\hline AST (U/L) & $27.4 \pm 3.0$ & $27.6 \pm 2.1$ & $28.2 \pm 2.4$ & $28.0 \pm 2.1$ & $27.3 \pm 1.4$ \\
\hline
\end{tabular}

Denote: ${ }^{*} \mathrm{P}<0.05$ versus other groups; ${ }^{\#} \mathrm{P}<0.05$ versus Atorvastatin and Combined groups; ${ }^{\circledR} \mathrm{P}<0.05$ versus Atorvastatin and Combined groups; ${ }^{*} \mathrm{P}<0.05$ versus Control, Atorvastatin and Colchicine groups.

Changes of NO production and serum level of Lp-PLA2 As presented in Table 2, after 6 weeks of high-fat and high-cholesterol diet administration, NO production in the hyperlipidemic model groups were significantly abolished when compared to the sham group, whereas serum levels of Lp-PLA2 were significantly elevated, indicating that hyperlipidemia might not only contribute to enhanced inflammation but also impaired endothelial function. After 2 weeks of treatment, NO production in the atorvastatin, colchicine and combined groups were increased when compared to the control group, and additionally the serum levels of Lp-PLA2 were concomitantly decreased. When

Table 2 Changes of NO production and serum level of Lp-PLA2

\begin{tabular}{|c|c|c|c|c|c|}
\hline Variables & Sham & Control & Atorvastatin & Colchicine & Combined \\
\hline \multicolumn{6}{|c|}{ Before the beginning of the study } \\
\hline $\mathrm{NO}(\mu \mathrm{mol} / \mathrm{L})$ & $8.6 \pm 0.7$ & $8.9 \pm 0.7$ & $9.2 \pm 0.5$ & $9.0 \pm 0.6$ & $8.8 \pm 0.6$ \\
\hline Lp-PLA2 (ng/mL) & $73.6 \pm 6.7$ & $78.7 \pm 7.3$ & $74.5 \pm 6.6$ & $76.2 \pm 6.9$ & $76.0 \pm 5.7$ \\
\hline \multicolumn{6}{|c|}{6 weeks of model production } \\
\hline $\mathrm{NO}(\mu \mathrm{mol} / \mathrm{L})$ & $8.2 \pm 0.4^{*}$ & $4.3 \pm 0.5$ & $4.1 \pm 0.4$ & $4.4 \pm 0.6$ & $4.5 \pm 0.6$ \\
\hline Lp-PLA2 (ng/mL) & $77.2 \pm 8.1^{*}$ & $164.2 \pm 13.5$ & $168.7 \pm 11.5$ & $170.3 \pm 15.2$ & $166.8 \pm 14.3$ \\
\hline \multicolumn{6}{|l|}{2 weeks of therapy } \\
\hline $\mathrm{NO}(\mu \mathrm{mol} / \mathrm{L})$ & $8.9 \pm 0.5$ & $4.4 \pm 0.6^{\#}$ & $6.2 \pm 0.7$ & $6.1 \pm 0.4$ & $7.3 \pm 0.3^{*}$ \\
\hline Lp-PLA2 (ng/mL) & $80.1 \pm 9.6$ & $160.6 \pm 10.2^{\#}$ & $148.3 \pm 9.9$ & $143.2 \pm 8.6$ & $130.2 \pm 5.8^{*}$ \\
\hline
\end{tabular}

Denote: ${ }^{*} \mathrm{P}<0.05$ versus other groups; ${ }^{\#} \mathrm{P}<0.05$ versus Atorvastatin, Colchicine and Combined groups; ${ }^{*} \mathrm{P}<0.05$ versus Control, Atorvastatin and Colchicine groups. 
compared to the atorvastatin group, the magnitude of LpPLA2 reduction was a little bit larger in the colchicine group, although there was no significant difference $(143.2 \pm 8.6 \mathrm{ng} / \mathrm{mL}$ versus $148.3 \pm 9.9 \mathrm{ng} / \mathrm{mL}, \mathrm{P}=0.063)$, and NO production between atorvastatin and colchicine groups was also comparable $(6.2 \pm 0.7 \mu \mathrm{mol} / \mathrm{L}$ versus $6.1 \pm$ $0.4 \mu \mathrm{mol} / \mathrm{L}, \mathrm{P}=0.106)$. Notably and interestingly, not only NO production was more profoundly increased, but LpPLA2 reduction was also more prominent in the combined group in comparison of the other hyperlipidemic groups, indicating that the combination of atorvastatin and colchicine had synergistic effects in rats with hyperlipidemia.

\section{Discussion}

Our current study shows that in rats with hyperlipidemia, an early stage of atherosclerosis, colchicine therapy alone is potential in simultaneously ameliorating inflammation and improving endothelial function, which is independent of lipid-lowering. The effects of CRP and Lp-PLA2 reduction and NO production are further enhanced when atorvastatin combined with colchicine therapy, indicating that these two drugs may have synergistic benefits on deterring atherogenesis and atherosclerotic progression.

Hyperlipidemia, which is characterized by increased LDL-C and TC levels and/or decreased HDL-C level, is a major risk factor for CVD. The underpinning mechanisms by which hyperlipidemia contributing to atherogenesis and atherosclerotic progression involve impairing endothelial function and eliciting endothelial activation, increasing foam cells formation, and enhancing vascular inflammation $[4,26,27]$. Therefore, treating hyperlipidemia with medications such as statins is favorable for ameliorating vascular inflammation, improving endothelial function and deterring atherosclerosis. As shown in our current study, in rats with hyperlipidemia producing by high-fat and highcholesterol diet, 2 weeks of atorvastatin $(10 \mathrm{mg} / \mathrm{kg} /$ day $)$ therapy significantly improved dyslipidemia and reduced CRP level, which was consistent with previous finding [9]. Expectedly, the traditional potent anti-inflammatory medicine colchicine also had a robust effect on declining serum CRP level, which was independent of lipid-lowering. As is well known that, increased CRP level is a significant risk factor for adverse cardiovascular outcomes as strongly supported by the JUPITER trial in which, when compared to placebo therapy, rosuvastatin significantly reduces adverse cardiovascular events in participants with normal lipid profile but with increased CRP level [28]. Our current study showed that the efficacy of statins on CRP reduction was further enhanced by colchicine addition suggesting that these two medications may have synergistic effects on improving systemic inflammation and may also have additional benefits for cardiovascular events prevention.

As is well documented that endothelial dysfunction, in terms of reduced NO production, plays critical roles on atherosclerosis initiation and progression [29], and additionally, as Lp-PLA2 elevation has been recognized as a new target for therapy in patients with CVD [30], we further investigated the effects of colchicine on endothelial function and Lp-PLA2 level. Previously, some studies have been conducted to evaluate the effects of statins on serum Lp-PLA2 level, and the results indicated that statins had modest effect on Lp-PLA2 reduction, and currently two large randomized controlled phase-III clinical trials (STABILITY and SOLID-TIMI 52) are ongoing to evaluate the efficacies of darapladib (a specific inhibitor for Lp-PLA2) on cardiovascular outcomes, which we believe may shift the paradigm of CVD therapy in the future due to the highly sensitive and specific characteristic of Lp-PLA2 for vascular inflammation. The results from our current study indicated that colchicine has a marginally better effect on Lp-PLA2 reduction than atorvastatin $(143.2 \pm 8.6 \mathrm{ng} / \mathrm{mL}$ versus $148.3 \pm 9.9 \mathrm{ng} / \mathrm{mL}, \mathrm{P}=0.063)$. In light of the mechanisms of actions of colchicine [31], we speculated that the efficacy of colchicine on Lp-PLA2 reduction might be at least partially associated with its effects on inhibiting inflammatory cells migration and infiltration. Since circulating Lp-PLA2 is largely produced by macrophages within vascular wall, therefore, inhibiting leukocytes adhesion and activation by colchicine was favorable for reducing Lp-PLA2 production. Additionally, increased NO production, which we considered derived from vascular inflammation amelioration, by colchicine therapy might reciprocally contribute to Lp-PLA2 production. Since NO could diminish oxidative stress and reduce ox-LDL production, which in turn leads to decrease foam cells formation and LpPLA2 excretion by macrophages and foam cells [29,32]. Taken together, we believed that colchicine reducing LpPLA2 production was dependent on its effects on ameliorating inflammation and improving endothelial function. Importantly, NO production and Lp-PLA2 reduction were more prominent in colchicine combined with atorvastatin therapy, indicating that adding colchicine to statins therapy might further enhance the protective effects of statins therapy. These mechanisms might at least partially explain the protective effect of statins combined with colchicine therapy on reducing cardiovascular events in patients with stable chronic coronary artery disease [23,33]. However, since the animal model of our current study was a simple scenario in terms of only having hyperlipidemia, whether colchicine really has an amazing and synergistic effect on more complicated conditions such as metabolism syndrome ensuing acute myocardial infarction in which endothelial function maybe already irreversible and inflammatory cascade within atherosclerotic plaque maybe already uncontrollable needs to be further investigated.

Finally, with regard to the potential side effects of colchicine combined with statins therapy, serum level of 
liver enzymes such ALT and AST were evaluated before and after therapy, and without any significant increase of liver enzymes was found. However, because our current study has not detected the changes of creatinine kinase levels, we cannot exclude the potential myopathy incidence induced by colchicine combined with statins therapy. Therefore, in the future to investigate whether colchicine combined with statins would increase the risk of myopathy is of particular importance.

\section{Conclusion}

Results from our current study show that in rats with hyperlipidemia, colchicine therapy is beneficial for reducing CRP level, increasing NO production and decreasing Lp-PLA2 level, which is independent of lipid-lowering. Colchicine combined with atorvastatin therapy has synergistic effects on improving endothelial function and ameliorating inflammation which we believe may be helpful and beneficial for future studies in exploring optimal therapeutic strategies for atherosclerosis and CVD preventions in the setting of hyperlipidemia.

\section{Competing interests}

The authors declare that they have no competing interests.

\section{Authors' contribution}

CC, CW and XD performed this study, HZ performed statistic analyses, and $\mathrm{CH}$ designed this study and wrote this article. All authors read and approved the final manuscript.

\section{Authors' information}

Congwu Huang and Chuan Cen co-first authors.

\section{Acknowledgement}

We appreciate very much for this work was supported by the grants from the Technology Project Foundation of Guangdong Province, China (2009B060700074 and 2010B060900079).

\section{Author details}

${ }^{1}$ The Department of Internal Medicine, the Second Affiliated Hospital of Shantou University, Shantou, China. ${ }^{2}$ The Department of Internal Medicine, Zhongshan People's Hospital, Zhongshan, China. ${ }^{3}$ The Emergency Department, the Second Affiliated Hospital of Dalian Medical University, Dalian, China.

Received: 17 March 2014 Accepted: 7 April 2014

Published: 17 April 2014

\section{References}

1. Montori VM, Brito JP, Ting HH: Patient-centered and practical application of new high cholesterol guidelines to prevent cardiovascular disease. JAMA 2014, 311:465-466.

2. Perk J, De Backer G, Gohlke H, Graham I, Reiner Z, Verschuren M, Albus C, Benlian P, Boysen G, Cifkova R, Deaton C, Ebrahim S, Fisher M, Germano G, Hobbs R, Hoes A, Karadeniz S, Mezzani A, Prescott E, Ryden L, Scherer M, Syvanne M, op Reimer WJ S, Vrints C, Wood D, Zamorano JL, Zannad F: European Guidelines on cardiovascular disease prevention in clinical practice (version 2012). The Fifth Joint Task Force of the European Society of Cardiology and Other Societies on Cardiovascular Disease Prevention in Clinical Practice (constituted by representatives of nine societies and by invited experts). Eur Heart J 2012, 33:1635-1701.

3. Domagala TB, Szeffler A, Dobrucki LW, Dropinski J, Polanski S, LeszczynskaWiloch M, Kotula-Horowitz K, Wojciechowski J, Wojnowski L, Szczeklik A, Kalinowski L: Nitric oxide production and endothelium-dependent vasorelaxation ameliorated by $\mathrm{N} 1$-methylnicotinamide in human blood vessels. Hypertension 2012, 59:825-832.

4. Xiao H, Lu M, Lin TY, Chen Z, Chen G, Wang WC, Marin T, Shentu TP, Wen L, Gongol B, Sun W, Liang X, Chen J, Huang HD, Pedra JH, Johnson DA, Shyy $J Y$ : Sterol regulatory element binding protein 2 activation of NLRP3 inflammasome in endothelium mediates hemodynamic-induced atherosclerosis susceptibility. Circulation 2013, 128:632-642.

5. Zechariah A, ElAli A, Hagemann N, Jin F, Doeppner TR, Helfrich I, Mies G, Hermann DM: Hyperlipidemia attenuates vascular endothelial growth factor-induced angiogenesis, impairs cerebral blood flow, and disturbs stroke recovery via decreased pericyte coverage of brain endothelial cells. Arterioscler Thromb Vasc Biol 2013, 33:1561-1567.

6. Puri R, Nissen SE, Libby P, Shao M, Ballantyne CM, Barter PJ, Chapman MJ, Erbel R, Raichlen JS, Uno K, Kataoka Y, Nicholls SJ: C-reactive protein, but not low-density lipoprotein cholesterol levels, associate with coronary atheroma regression and cardiovascular events after maximally intensive statin therapy. Circulation 2013, 128:2395-2403.

7. Sever PS, Poulter NR, Chang CL, Thom SA, Hughes AD, Welsh P, Sattar N: Evaluation of C-reactive protein before and on-treatment as a predictor of benefit of atorvastatin: a cohort analysis from the Anglo-Scandinavian Cardiac Outcomes Trial lipid-lowering arm. J Am Coll Cardiol 2013, 62:717-729.

8. Cai A, Zheng D, Dong Y, Qiu R, Huang Y, Song Y, Jiang Z, Rao S, Liao X, Kuang J, Dai G, Mai W: Efficacy of Atorvastatin combined with adiposederived mesenchymal stem cell transplantation on cardiac function in rats with acute myocardial infarction. Acta Biochim Biophys Sin (Shanghai) 2011, 43:857-866.

9. Ott C, Schneider MP, Schlaich MP, Schmieder RE: Rosuvastatin improves pulse wave reflection by restoring endothelial function. Microvasc Res 2012, 84:60-64.

10. Toth PP: Making a case for quantitative assessment of cardiovascular risk. J Clin Lipidol 2007, 1:234-241.

11. Turunen P, Puhakka H, Rutanen J, Hiltunen MO, Heikura T, Gruchala M, Yla-Herttuala S: Intravascular adenovirus-mediated lipoprotein-associated phospholipase A2 gene transfer reduces neointima formation in balloon-denuded rabbit aorta. Atherosclerosis 2005, 179:27-33.

12. Constantinos A, Demopoulos Haralabos C, Karantonis Smaragdi A: Platelet activating factor-a molecular link between atherosclerosis theories. Eur $\mathrm{J}$ Lipid Sci Tech 2003, 105:705-716.

13. Garza CA, Montori VM, McConnell JP, Somers VK, Kullo IJ, Lopez-Jimenez F: Association between lipoprotein-associated phospholipase A2 and cardiovascular disease: a systematic review. Mayo Clin Proc 2007, 82:159-165.

14. Wilensky RL, Shi Y, Mohler ER 3rd, Hamamdzic D, Burgert ME, Li J, Postle A, Fenning RS, Bollinger JG, Hoffman BE, Pelchovitz DJ, Yang J, Mirabile RC, Webb CL, Zhang L, Zhang P, Gelb MH, Walker MC, Zalewski A, Macphee $\mathrm{CH}$ : Inhibition of lipoprotein-associated phospholipase A2 reduces complex coronary atherosclerotic plaque development. Nat Med 2008, 14:1059-1066.

15. Daniels LB, Laughlin GA, Sarno MJ, Bettencourt R, Wolfert RL, Barrett-Connor E: Lipoprotein-associated phospholipase A2 is an independent predictor of incident coronary heart disease in an apparently healthy older population: the Rancho Bernardo Study. J Am Coll Cardiol 2008, 51:913-919.

16. Epps KC, Wilensky RL: Lp-PLA(2)- a novel risk factor for high-risk coronary and carotid artery disease. J Intern Med 2011, 269:94-106.

17. Cai A, Zheng D, Qiu R, Mai W, Zhou Y: Lipoprotein-associated phospholipase A2 (Lp-PLA(2)): a novel and promising biomarker for cardiovascular risks assessment. Dis Markers 2013, 34:323-331.

18. Song JX, Ren JY, Chen H: Simvastatin reduces lipoprotein-associated phospholipase A2 in lipopolysaccharide-stimulated human monocytederived macrophages through inhibition of the mevalonategeranylgeranyl pyrophosphate-RhoA-p38 mitogen-activated protein kinase pathway. J Cardiovasc Pharmacol 2011, 57:213-222.

19. Tsantila N, Tsoupras AB, Fragopoulou E, Antonopoulou S, latrou C, Demopoulos CA: In vitro and in vivo effects of statins on plateletactivating factor and its metabolism. Angiology 2011, 62:209-218.

20. Lee SH, Kang SM, Park S, Jang Y, Chung N, Choi D: The effects of statin monotherapy and low-dose statin/ezetimibe on lipoprotein-associated phospholipase A(2). Clin Cardiol 2011, 34:108-112.

21. Beltowski J, Wojcicka G, Jamroz A: Effect of 3-hydroxy-3-methylglutarylcoenzyme A reductase inhibitors (statins) on tissue paraoxonase 1 and plasma platelet activating factor acetylhydrolase activities. J Cardiovasc Pharmacol 2004, 43:121-127. 
22. Macphee $\mathrm{CH}$, Nelson JJ, Zalewski A: Lipoprotein-associated phospholipase A2 as a target of therapy. Curr Opin Lipidol 2005, 16:442-446.

23. Nidorf SM, Eikelboom JW, Budgeon CA, Thompson PL: Low-dose colchicine for secondary prevention of cardiovascular disease. J Am Coll Cardiol 2013, 61:404-410.

24. Modschiedler K, Weller M, Worl P, Den Driesch PV: Dapsone and colchicine inhibit adhesion of neutrophilic granulocytes to epidermal sections. Arch Dermatol Res 2000, 292:32-36.

25. Yang YH, Yang J, Jiang QH: Hypolipidemic effect of gypenosides in experimentally induced hypercholesterolemic rats. Lipids Health Dis 2013, 12:154.

26. Schlager $O$, Widhalm K, Hammer A, Giurgea A, Margeta C, Fritsch M, Zehetmayer S, Koppensteiner R, Gschwandtner ME, Willfort-Ehringer A: Familial hypercholesterolemia affects microvascular autoregulation in children. Metabolism 2013, 62:820-827.

27. Ahmed M, El Bakly WM, Zaki AM, Abd ALF, El SO: Bupropion effects on high-fat diet-induced steatohepatitis and endothelial dysfunction in rats: role of tumour necrosis factor-alpha.LID. J Pharm Pharmacol 2014, doi:10.1111/jphp.12213.

28. Ridker PM, Danielson E, Fonseca FA, Genest J, Gotto AM Jr, Kastelein JJ, Koenig W, Libby P, Lorenzatti AJ, MacFadyen JG, Nordestgaard BG, Shepherd J, Willerson JT, Glynn RJ: Rosuvastatin to prevent vascular events in men and women with elevated C-reactive protein. $\mathrm{N}$ Engl J Med 2008, 359:2195-2207.

29. Liao JK: Linking endothelial dysfunction with endothelial cell activation. J Clin Invest 2013, 123:540-541

30. Macphee $\mathrm{CH}$, Nelson J, Zalewski A: Role of lipoprotein-associated phospholipase A2 in atherosclerosis and its potential as a therapeutic target. Curr Opin Pharmacol 2006, 6:154-161.

31. Lu Y, Chen J, Xiao M, Li W, Miller DD: An overview of tubulin inhibitors that interact with the colchicine binding site. Pharm Res 2012, 29:2943-2971.

32. Forstermann $U$, Munzel $\mathrm{T}$ : Endothelial nitric oxide synthase in vascular disease: from marvel to menace. Circulation 2006, 113:1708-1714.

33. Nidorf $\mathrm{M}$, Thompson PL: Effect of colchicine ( $0.5 \mathrm{mg}$ twice daily) on highsensitivity C-reactive protein independent of aspirin and atorvastatin in patients with stable coronary artery disease. Am J Cardiol 2007, 99:805-807.

doi:10.1186/1476-511X-13-67

Cite this article as: Huang et al:: Synergistic effects of colchicine combined with atorvastatin in rats with hyperlipidemia. Lipids in Health and Disease 2014 13:67

\section{Submit your next manuscript to BioMed Central and take full advantage of:}

- Convenient online submission

- Thorough peer review

- No space constraints or color figure charges

- Immediate publication on acceptance

- Inclusion in PubMed, CAS, Scopus and Google Scholar

- Research which is freely available for redistribution 